\title{
Identification of Pisum sativum Germ Plasm with Resistance to Root Rot Caused by Multiple Strains of Aphanomyces euteiches
}

\author{
D. K. Malvick and J. A. Percich, Department of Plant Pathology, University of Minnesota-Twin Cities, St. Paul \\ 55108
}

\begin{abstract}
Malvick, D. K., and Percich, J. A. 1999. Identification of Pisum sativum germ plasm with resistance to root rot caused by multiple strains of Aphanomyces euteiches. Plant Dis. 83:51-54.

Aphanomyces root rot is a serious disease of pea (Pisum sativum), and additional sources of resistance are needed for development of disease-resistant cultivars. Accessions $(n=123)$ from the P. sativum Plant Introduction (PI) collection with the highest relative levels of resistance to one strain of Aphanomyces euteiches were previously identified from among approximately 2,500 accessions evaluated. The chosen 123 accessions were evaluated in this study for resistance to root rot caused by multiple strains of this pathogen. Five strains representing different US geographical locations and pathogenicity characteristics were used to evaluate pea seedlings in a greenhouse. Disease severity (DS) and percent loss of fresh biomass (inoculated vs. noninoculated plants) were determined 15 days after inoculation. Significant differences $(P=0.05)$ in levels of DS and biomass loss (BL) occurred among the accessions after inoculation individually with the five strains. The relative rank of accessions based on DS and BL varied with the strain of A. euteiches used for inoculations. The 20 accessions with the lowest DS after inoculation with each strain were identified. Based on lowest DS, two accessions were among the 20 identified with all five individual strains, and four other accessions were among the 20 identified with four of the five strains. The results suggest that the $P$. sativum PI collection contains useful accessions for breeding programs aimed at developing pea varieties with resistance to $A$. euteiches.
\end{abstract}

Additional keywords: legume, Oomycota, pea

Pea (Pisum sativum L.) is widely grown throughout the world. In the United States, pea production for the canning and freezing industry is focused in the Midwest and Pacific Northwest. Disease can be a severe limiting factor to production in all areas where peas are cultivated. One serious disease of pea is Aphanomyces root rot caused by Aphanomyces euteiches Drechs. $(6,8,16)$. A . euteiches is a soilborne organism classified in Kingdom Chromista, Division Oomycota. (15). Aphanomyces root rot occurs in many geographical regions, including Europe, Australia, Japan, Canada, and the United States (16). Persson et al. (17) reported that $A$. euteiches was the most yield-reducing pathogen of pea in Denmark and southern

Corresponding author: D. K. Malvick

E-mail: dmalvick@inwave.com

Current address: W-L Research, Inc.; 8701 West U.S. Highway 14; Evansville, WI 53536.

Published as contribution No. 191220026 of the series of the Minnesota Agricultural Experiment Station. This project was supported by funding provided by the USDA Cool Season Food Legume Research Program, project proposal no. 9607711.

Accepted for publication 2 October 1998.

Publication no. D-1998-1113-01R

(C) 1999 The American Phytopathological Society
Sweden. Average annual yield losses of pea due to $A$. euteiches are estimated at $10 \%$ in the Midwestern United States (6). Entire fields, however, are frequently destroyed during wet years.

Aphanomyces root rot has remained a destructive disease of pea for over 70 years (8) due to ineffective disease control methods. Crop rotation, host resistance, fungicides, and biological control agents have been investigated to manage this disease $(6,16)$. These tactics have shown promise, but have not provided an adequate level of control for commercial production. Disease is reduced primarily through crop rotation and avoidance of fields highly infested with A. euteiches. Varieties with resistance to Aphanomyces root rot may be a key component in an integrated strategy for disease control. Several pea breeding lines have been developed that express resistance to Aphanomyces root rot $(2,5,9)$; however, their resistance traits have not been incorporated into commercial cultivars.

Aphanomyces root rot resistance in pea has been difficult to achieve due to challenges in identifying and incorporating useful disease resistance traits, and because of pathogenic variability in populations of A. euteiches $(1,4,11,13)$. Variability within populations of $A$. euteiches may cause inconsistent performance of disease-resistant pea lines in the field, and could influ- ence the durability and effectiveness of resistance in different geographical areas $(4,11,13,19)$. Breeding for resistance to Aphanomyces root rot may be enhanced if additional resistance traits that are effective against a variety of distinct strains of $A$. euteiches are identified. Few disease resistance genes in pea appear to be available to breeders. The United States Department of Agriculture (USDA) P. sativum Plant Introduction (PI) collection may be a source of resistance genes. The first extensive study of Aphanomyces root rot resistance in pea accessions was published in 1960 (10). In a recent study, 2,500 accessions, comprising most of the PI collection, were screened for resistance to Aphanomyces root rot using one isolate of $A$. euteiches (12).

The aim of this study was to identify the best sources of resistance to Aphanomyces root rot in the P. sativum PI collection. The 123 accessions evaluated in this study were previously identified to be those with the highest relative level of disease resistance in the PI collection (12). Our primary objective was to determine how the 123 accessions interact with five strains of $A$. euteiches that represent different pathogenicity characteristics and geographical areas.

\section{MATERIALS AND METHODS}

Strains of A. euteiches. Five strains of A. euteiches (Table 1) from different geographical areas with differing pathogenicity characteristics on pea, alfalfa, and bean were included in this study $(11,13)$. Strains were characterized as A. euteiches according to the keys of Dick (3) and Scott (18) Strains of $A$. euteiches were isolated from soil by baiting with $P$. sativum seedlings (cv. Perfection 8221), and single zoospore cultures were prepared as described previously (11). All strains used for inoculations except Ae467 originated from single zoospore cysts. Cultures were maintained on corn meal agar (Difco Laboratories, Detroit) and stored at $4{ }^{\circ} \mathrm{C}$.

$P$. sativum accessions evaluated. $P$. sativum accessions $(n=123)$ from the USDA Western Regional Plant Introduction Station in Pullman, Washington were evaluated (Table 2). These represent the best $5 \%$ of all available accessions based on percent loss of fresh biomass (inoculated vs. noninoculated plants) after inoculation with strain Ae467 of A. euteiches (12). Additional information on 
these and other $P$. sativum accessions is available in the USDA Germplasm Resources Information Network.

Pathogenicity studies. The 123 P. sativum accessions were inoculated separately with the five strains of A. euteiches. Pea cultivar Little Marvel was the susceptible control, and breeding lines MN313, MN314, and WI8904 were included as resistant controls $(2,5)$. Waxed paper cups
(160 ml) containing vermiculite were sown with three or four seeds. Plants were grown in a greenhouse with a 12-h photoperiod and fertilized with $0.5 \times$ Hoagland's solution (7) 1 week after inoculation. Temperature was maintained at $23 \pm 2^{\circ} \mathrm{C}$. Zoospores were produced as described by Mitchell and Yang (14), counted with a hemacytometer, and adjusted to 500 zoospores $/ \mathrm{ml}$. The inoculum $(5 \mathrm{ml})$ was in-

Table 1. Strains of Aphanomyces euteiches used for evaluation of resistance to Aphanomyces root rot in pea

Pathogenicity to five pea germ plasm lines ${ }^{\mathrm{a}, \mathrm{b}}$

\begin{tabular}{lcccccc}
\cline { 2 - 6 } Strain & Geographical origin & MN313 & MN314 & 90-2079 & WI8904 & Little Marvel \\
\hline Ae467 & Wisconsin & - & - & - & + & + \\
AeMM183 (1) & Minnesota & - & - & - & - & + \\
AeMM62 (3) & Minnesota & - & - & + & + & + \\
AeOR5 (2) & Oregon & + & + & + & + & + \\
P134 (1) & Wisconsin & + & + & + & + & + \\
\hline
\end{tabular}

a + denotes the strain incites disease severity $>3$ on a particular host based on severity of root infection, where $0=$ a healthy plant with no symptoms and $5=$ a dead plant.

${ }^{\mathrm{b}}$ MN313 and MN314 are resistant pea breeding lines (2); 90-2079 is a moderately resistant breeding line (9); WI8904 is a moderately resistant breeding line (5); and Little Marvel is a susceptible cultivar.

Table 2. Pisum sativum PI accessions evaluated for resistance against five strains of Aphanomyces euteiches

\begin{tabular}{ccccc}
\hline Accession & Accession & Accession & Accession & Accession \\
\hline 102887 & 196015 & 261613 & 347410 & 471190 \\
116056 & 196023 & 269794 & 356982 & 471195 \\
116944 & 206798 & 269802 & 357003 & 471314 \\
143486 & 206811 & 272215 & 357006 & 471331 \\
162909 & 206816 & 272216 & 357030 & 471336 \\
164396 & 206824 & 272217 & 358612 & 471354 \\
164669 & 210613 & 273681 & 358627 & 471357 \\
165965 & 210641 & 275639 & 358630 & 471387 \\
169600 & 210642 & 280608 & 358640 & 471388 \\
170669 & 210678 & 286607 & 358667 & 471409 \\
173058 & 210684 & 306592 & 358679 & 471410 \\
174321 & 226562 & 312200 & 358693 & 471438 \\
176721 & 244140 & 314798 & 365424 & 494077 \\
179019 & 244141 & 320973 & 390795 & 494506 \\
180471 & 244144 & 324702 & 390808 & 494508 \\
181800 & 244151 & 324705 & 393487 & 494510 \\
183910 & 244154 & 343267 & 411143 & 494511 \\
185183 & 244158 & 343293 & 413686 & 494515 \\
193579 & 244160 & 343958 & 413696 & 505080 \\
193586 & 244162 & 343984 & 413703 & 505102 \\
193589 & 244163 & 347284 & 429843 & 505133 \\
193835 & 244184 & 347299 & 471114 & 512078 \\
193846 & 244226 & 347301 & 471123 & 512087 \\
193849 & 244259 & 347304 & 471128 & \\
195629 & 257593 & 347327 & 471166 & \\
\hline
\end{tabular}

Table 3. Percentage of 123 Plant Introduction accessions tested within various disease severity (DS) and percent biomass loss (BL) classes after inoculation individually with five strains of Aphanomyces euteiches $^{\mathrm{a}}$

\begin{tabular}{lccccc}
\hline & \multicolumn{3}{c}{ DS class $^{\mathbf{b}}$} & & \multicolumn{2}{c}{ BL class (\%) $^{\mathbf{c}}$} \\
\cline { 2 - 3 } \cline { 5 - 6 } Strain & $\mathbf{0 - 2 . 9}$ & $\mathbf{3 . 0 - 5 . 0}$ & & $\mathbf{0 - 5 0}$ & $\mathbf{5 0 - 1 0 0}$ \\
\hline Ae467 & 8 & 92 & & 52 & 48 \\
AeMM183(1) & 3 & 97 & & 19 & 81 \\
AeMM62(3) & 2 & 98 & & 9 & 91 \\
AeOR5(2) & 11 & 89 & & 65 & 35 \\
P134(1) & 6 & 94 & & 63 & 37 \\
\hline
\end{tabular}

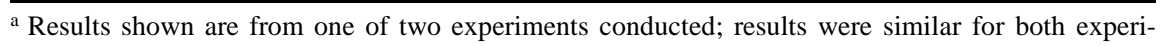
ments.

${ }^{\mathrm{b}}$ DS is based on severity of root infection, where $0=$ a healthy plant with no symptoms and $5=\mathrm{a}$ dead plant. DS > 3.0 indicates a clear pathogenic interaction.

${ }^{\mathrm{c}}$ Percent loss of biomass is based on fresh biomass of inoculated vs. noninoculated plants.

jected approximately $1 \mathrm{~cm}$ below the vermiculite surface next to seedlings 7 days after planting. Vermiculite in cups was saturated at time of inoculation and watered thereafter as needed to keep vermiculite moist. At 15 days after inoculation, the plants were washed and disease severity (DS) and fresh biomass were determined. DS was based on a 0 to 5 scale, where $0=$ no macroscopic symptoms; $1=$ a few small discolored lesions on roots; $2=$ minor discoloration covering less than $90 \%$ of the root system; 3 = over $90 \%$ of root system brown or yellowed, but no symptoms on epicotyl or hypocotyl; $4=$ all of root system brown or soft and epicotyl or hypocotyl shriveled or brown; and $5=$ plant dead (2). Percent loss of biomass was based on fresh biomass of equal numbers of inoculated and noninoculated plants for each accession. Three replicate cups were used for noninoculated controls and for each combination of strain and plant host. The experiment was organized as a completely randomized design. The experiment was repeated once. DS and biomass loss (BL) values among all accessions inoculated with individual strains were subjected to analysis of variance and means were compared with Fisher's protected least significant difference test (SAS software, SAS Institute, Inc., Cary, NC).

\section{RESULTS}

Significant differences in resistance to Aphanomyces root rot were identified among the 123 accessions tested, although most were clearly susceptible. Over $88 \%$ of the 123 P. sativum accessions were susceptible (DS > 2.9) to each of the five strains of A. euteiches (Table 3). Fresh BL of most $(75 \%)$ accessions was $>30 \%$ after inoculation with each strain (data not shown). The DS and percent BL of accessions differed significantly $(P=0.05)$ after inoculation with the five strains. All 123 accessions were ranked from low to high DS and BL based on the five individual strains and the average across all strains. The 20 accessions with the lowest mean DS based on inoculation with each strain in two replicate experiments are listed in Table 4. The 20 accessions with the lowest DS and BL based on the mean DS and BL values from all strains combined in both experiments are shown in Table 5. Approximately $50 \%$ of the top 20 accessions were significantly better than the highly susceptible cultivar Little Marvel and the moderately resistant breeding line WI8904. The highly resistant breeding lines MN313 and MN314 expressed lower DS and BL than at least $50 \%$ of the top 20 accessions. The noninoculated control plants remained free of root rot symptoms in all replications and experiments.

The $P$. sativum accessions reacted differently with the five strains of A. euteiches. Individual strains caused significantly different $(P=0.05)$ DS and BL on all acces- 
sions except 411143, 413696, and 512078. Results were similar for both experiments. The top 20 accessions ranked by lowest DS and BL differed for the five strains (Table 4). Two accessions appeared on all five of the ranked lists based on DS (244162 and 393487), and four (210641, 269802, 411143, and 413696) appeared on four lists. One accession (393487) appeared on five ranked lists based on BL, and two accessions (244226 and 413696) appeared on four lists. Many of the best 20 accessions identified with individual strains differed from the best 20 identified with averages of DS and BL across all strains.

\section{DISCUSSION}

The main purpose of this study was to identify the $P$. sativum accessions from the PI collection that are most likely to be useful as sources of resistance to $A$. euteiches. Increasing the levels of resistance in pea to A. euteiches may be the most desirable approach to control of Aphanomyces root rot. P. sativum accessions were identified that may have good potential for use in pea breeding programs designed to improve resistance to Aphanomyces root rot.

Most accessions from the 123 evaluated in the present study were highly susceptible to Aphanomyces root rot. Some accessions had significantly lower levels of DS, $\mathrm{BL}$, or both than the susceptible cultivar Little Marvel. Some of the best accessions were rated comparable to the Aphanomy- ces root rot-resistant pea breeding lines WI8904, MN313, and MN314 using the seedling test conducted in vermiculite under conditions that may favor the pathogen.
Performance of accessions may differ if they were allowed to grow over a longer time period, or were evaluated in soil under different environmental conditions.

Table 5. Accessions of Pisum sativum $(n=20)$ with the lowest mean disease severity (DS) and percent fresh biomass loss (BL) based on evaluation individually with five strains of Aphanomyces euteiches $^{\mathrm{a}}$

\begin{tabular}{llll}
\hline Accession & DS $^{\mathbf{b}}$ & Accession & BL $(\boldsymbol{\%})^{\mathbf{c}}$ \\
\hline 210641 & 2.1 & 244162 & 17 \\
411143 & 2.4 & 269802 & 22 \\
210642 & 2.5 & 393487 & 23 \\
393487 & 2.5 & 413696 & 24 \\
244162 & 2.6 & 390808 & 26 \\
269802 & 2.8 & 471354 & 26 \\
413696 & 2.9 & 343984 & 28 \\
343984 & 3.0 & 210642 & 29 \\
505102 & 3.2 & 210641 & 29 \\
494506 & 3.2 & 244226 & 29 \\
512078 & 3.2 & 411143 & 29 \\
286607 & 3.2 & 365424 & 30 \\
471354 & 3.3 & 185183 & 30 \\
324702 & 3.3 & 196015 & 30 \\
471190 & 3.3 & 471409 & 31 \\
358679 & 3.3 & 471166 & 33 \\
165965 & 3.4 & 343958 & 34 \\
272217 & 3.4 & 320973 & 34 \\
494508 & 3.4 & 358630 & 34 \\
206824 & 3.4 & 210684 & 34 \\
MN313 & 2.2 & MN313 & 21 \\
MN314 & 1.7 & MN314 & 13 \\
WI8904 & 3.8 & Wittle Marvel & 46 \\
Little Marvel & 4.5 & & 58 \\
\hline
\end{tabular}

${ }^{a}$ Results are the combined mean from all inoculations with five individual strains in two experiments.

b DS is based on severity of root infection, where $0=$ a healthy plant with no symptoms and $5=\mathrm{a}$ dead plant. DS > 3.0 indicates a clear pathogenic interaction.

c Percent loss of biomass is based on fresh biomass of inoculated vs. noninoculated plants

Table 4. Pisum sativum accessions ( $n=20$ per strain) with the lowest mean disease severity (DS) ratings based on individual evaluations with five strains of Aphanomyces euteiches ${ }^{\mathrm{a}}$

\begin{tabular}{|c|c|c|c|c|c|c|c|c|c|}
\hline \multicolumn{2}{|c|}{ Strain Ae467 } & \multicolumn{2}{|c|}{ Strain AeMM183 (1) } & \multicolumn{2}{|c|}{ Strain AeMM62 (3) } & \multicolumn{2}{|c|}{ Strain AeOR5 (2) } & \multicolumn{2}{|c|}{ Strain P134 (1) } \\
\hline Accession & $\mathbf{D S}^{\mathbf{b}}$ & Accession & DS & Accession & DS & Accession & DS & Accession & DS \\
\hline 210641 & 0.9 & 210641 & 0.2 & 210642 & 1.1 & 411143 & 0.1 & 393487 & 0.2 \\
\hline 210642 & 0.4 & 411143 & 2.3 & 210641 & 0.9 & 413696 & 0.5 & 324705 & 4.0 \\
\hline 286607 & 1.4 & 210642 & 0.9 & 244162 & 2.4 & 272217 & 1.1 & 411143 & 1.8 \\
\hline 269802 & 2.2 & 244162 & 2.3 & 393487 & 1.8 & 471190 & 0.1 & 471354 & 2.4 \\
\hline 343984 & 2.3 & 275639 & 0.5 & 471438 & 1.1 & 505102 & 0.8 & 494077 & 3.9 \\
\hline 210613 & 3.3 & 269802 & 1.8 & 324702 & 1.5 & 471195 & 0.1 & 505080 & 1.6 \\
\hline 494508 & 2.9 & 393487 & 2.3 & 269794 & 2.1 & 393487 & 3.2 & 413696 & 3.8 \\
\hline 393487 & 2.7 & 494506 & 2.8 & 165965 & 3.0 & 244151 & 1.3 & 244184 & 2.4 \\
\hline 244162 & 2.9 & 413696 & 1.7 & 471409 & 2.3 & 358693 & 0.3 & 505102 & 3.4 \\
\hline 471410 & 2.2 & 343984 & 2.1 & 494510 & 2.8 & 269802 & 1.8 & 226562 & 3.2 \\
\hline 273681 & 2.2 & 206824 & 2.1 & 471357 & 2.5 & 358679 & 1.3 & 244162 & 2.4 \\
\hline 358679 & 2.1 & 343958 & 2.0 & 347284 & 2.6 & 173058 & 0.1 & 206798 & 3.3 \\
\hline 164669 & 2.3 & 471357 & 2.5 & 269802 & 3.0 & 244163 & 0.2 & 356982 & 3.6 \\
\hline 343598 & 2.8 & 505133 & 2.5 & 41143 & 2.0 & 494506 & 1.0 & 494510 & 2.8 \\
\hline 471331 & 2.8 & 471123 & 1.8 & 413696 & 3.0 & 512078 & $\mathrm{NT}^{\mathrm{c}}$ & 324702 & 4.1 \\
\hline 358667 & 2.9 & 244144 & 1.6 & 512078 & 3.0 & 471354 & 2.3 & 244141 & 2.4 \\
\hline 471387 & 1.9 & 505102 & 2.3 & 273681 & 2.2 & 244162 & 2.0 & 210641 & 2.4 \\
\hline 471409 & 2.2 & 320973 & 2.7 & 365424 & 2.6 & 165965 & 1.1 & 358693 & 2.2 \\
\hline 244151 & 4.1 & 244226 & 2.4 & 181800 & 2.8 & 471410 & 0.8 & 471123 & 4.3 \\
\hline 494515 & 2.8 & 494515 & 2.8 & 179019 & 3.2 & 206824 & 2.4 & 512078 & 3.3 \\
\hline MN313 & 1.6 & MN313 & 1.9 & MN313 & 1.1 & MN313 & 3.6 & MN313 & 2.2 \\
\hline MN314 & 1.1 & MN314 & 0.3 & MN314 & 1.3 & MN314 & 2.0 & MN314 & 3.6 \\
\hline WI8904 & 4.6 & WI8904 & 4.8 & WI8904 & 3.9 & WI8904 & 3.5 & WI8904 & 1.7 \\
\hline Little Marvel & 4.4 & Little Marvel & 4.5 & Little Marvel & 4.7 & Little Marvel & 4.0 & Little Marvel & 4.1 \\
\hline LSD $^{d}$ & 1.4 & LSD & 1.6 & LSD & 1.3 & LSD & 2.3 & LSD & 1.3 \\
\hline
\end{tabular}

${ }^{a}$ Rankings for individual strains are based on the mean of DS values from two experiments. The DS values shown are for one of the two experiments; however, results were similar for both experiments.

${ }^{b}$ DS is based on severity of root infection, where $0=$ a healthy plant with no symptoms and $5=$ a dead plant.

${ }^{\mathrm{c}} \mathrm{NT}=$ not tested in this experiment, but was tested in replicate experiment.

${ }^{\mathrm{d}} \mathrm{LSD}=$ least significant difference. 
Regardless of these experimental considerations, the results suggest that some of the accessions identified may have relatively good potential for inclusion into breeding programs.

Five strains of the pathogen were shown to interact differently with the accessions. This demonstrates that pathogen isolate and strain selection can significantly affect the response of germ plasm to Aphanomyces root rot. The $P$. sativum accessions expressed different DS and BL after inoculation with the five strains of $A$. euteiches. These results suggest that minimal incorporation of pathogenic variability into breeding programs due to use of single isolates or single nurseries may have slowed progress in breeding for resistance. The overall best accessions may be those that have the relatively lowest DS and BL after inoculation with each of several different strains. Alternatively, the best accessions for breeding programs may be identified by combining the results from evaluation individually with a set of strains, and then ranking the pooled mean results. Another approach would be to use an inoculum "cocktail" consisting of a mixture of strains with different pathogenicity characteristics.

Inoculation with different strains may reveal various sources of resistance to Aphanomyces root rot. Accessions with dissimilar DS or BL after inoculation may demonstrate distinct interactions with unknown virulence factors in the different strains. Little is known of the traits controlling resistance to Aphanomyces root rot in $P$. sativum, and even less is known about the virulence and pathogenicity factors that are important in A. euteiches. The use of different pea accessions and strains of $A$. euteiches may be useful in studies delving into these questions. These results also illustrate the potential existence of strains with different pathogenicity and virulence characteristics in different geographical areas and, furthermore, a corresponding need for different sources of resistance. Various sources of resistance in pea to Aphanomyces root rot may not be effective or stable against predominant strains of $A$. euteiches present in different parts of the United States.

This study reveals challenges and promise in understanding and controlling Aphanomyces root rot of pea. Some accessions in the $P$. sativum PI collection appear to have useful traits for resistance to $A$. euteiches; however, much work is required to determine how useful the traits may be. Significant challenges remain in developing commercially acceptable pea cultivars with resistance to A. euteiches. Continued study of disease resistance traits available in $P$. sativum germ plasm and interactions between germ plasm and distinct strains may lead to the development of pea cultivars with improved resistance to Aphanomyces root rot.

\section{ACKNOWLEDGMENTS}

We thank C. Simon at the USDA-ARS Western Regional Plant Introduction Station for providing seed of the $P$. sativum accessions used in this study.

\section{LITERATURE CITED}

1. Beute, M. K., and Lockwood, J. L. 1967. Pathogenic variability in Aphanomyces euteiches. Phytopathology 57:57-60.

2. Davis, D. W., Fritz, V. A., Pfleger, F. L., Percich, J. A., and Malvick., D. K. 1995. MN 144, MN 313, and MN 314: Garden pea lines resistant to root rot caused by Aphanomyces euteiches Drechs. HortScience 30:639-640.

3. Dick, M. W. 1973. Saprolegniales. Pages 113144 in: The Fungi: An Advanced Treatise. Vol. IB. G. C. Ainsworth, F. K. Sparrow, and A. S. Sussman, eds. Academic Press, Orlando, FL.

4. Grau, C. R., Muehlchen, A. M., Tofte, J. E., and Smith, R. R. 1991. Variability in virulence of Aphanomyces euteiches. Plant Dis. 75:1153-1156.

5. Gritton, E. T. 1992. Registration of five root rot resistant germplasm lines of processing pea. Crop Sci. 30:1166-1167.

6. Hagedorn, D. J., ed. 1984. Compendium of Pea Diseases. The Am. Phytopathol. Soc. St. Paul, MN.

7. Hoagland, D. R., and Arnon, D. I. 1938. The water-culture method for growing plants without soil. Univ. Calif. Berkely Agric. Exp. Sta. Circ. 347:1-32.

8. Jones, F. R., and Drechsler, C. 1925. Root rot of peas in the United States caused by Aphanomyces euteiches. J. Agric. Res. 30:293-325.

9. Kraft, J. M. 1992. Registration of 90-2079, 90-2131, and 90-2322 pea germplasms. Crop Sci. 32:1076.

10. Lockwood, J. L. 1960. Pea introductions with partial resistance to Aphanomyces root rot. Phytopathology 50:621-624.

11. Malvick, D. K., Grau, C.R., and Percich, J. A. 1998. Characterization of Aphanomyces euteiches strains based on pathogenicity and random amplified polymorphic DNA analysis. Mycol. Res. 102:465-475.

12. Malvick, D. K., and Percich, J. A. 1996. Screening of pea plant introduction collection for resistance to Aphanomyces root rot. Biol. Cult. Tests 11:112.

13. Malvick, D. K., and Percich, J. A 1998. Genotypic and pathogenic diversity among pea-infecting strains of Aphanomyces euteiches from the central and western United States. Phytopathology 88:915-921.

14. Mitchell, J. E., and Yang, C. Y. 1966. Factors affecting growth and development of Aphanomyces euteiches. Phytopathology 56:917922.

15. Moore-Landecker, E. 1996. Fundamentals of the Fungi. Fourth Edition. Prentice hall. Upper Saddle River, New Jersey.

16. Papavizas, G. C., and Ayers, W. A. 1974. Aphanomyces species and their root diseases in pea and sugarbeet. U. S. Dep. Agric. Agric. Res. Serv. Tech. Bull. 1485.

17. Persson, L., Bodker, L., and LarssonWikstrom, M. 1997. Prevalence and pathogenicity of foot and root rot pathogens of pea in southern Scandinavia. Plant Dis. 81:171174.

18. Scott, W. W. 1961. A monograph of the genus Aphanomyces. Va. Agric. Exp. Stn. Tech. Bull. 151.

19. Sundheim, L. 1972. Physiologic specialization in Aphanomyces euteiches. Physiol. Plant Pathol. 2:301-306. 